\title{
Updates in the Management of CLL/SLL: Management of Relapsed/Refractory Disease and the Role of Minimal Residual Disease
}

\author{
Presented by Jennifer R. Brown, MD, PhD
}

\begin{abstract}
In relapsed chronic lymphocytic leukemia (CLL), the choice of therapy depends on the risk profile, prior therapy, and patient comorbidities. The first novel agent for patients who had previously received chemoimmunotherapy is typically a BTK inhibitor or combination venetoclax + rituximab. For patients with problematic comorbidities, however, a PI3K inhibitor can be used, but generally this is reserved for later lines of therapy. For those who stop ibrutinib due to adverse events, there are broad options but data are still limited. For those whose disease progresses on a BTK inhibitor, the only prospective data are for use of venetoclax. For patients who have been treated with venetoclax + rituximab and experience relapse, retreatment is a possibility if they have had a durable remission. Finally, undetectable minimal residual disease is strongly predictive of durability of response for time-limited regimens but not for continuous BTK inhibition, and is generally not indicated for monitoring patients.
\end{abstract}

J Natl Compr Canc Netw 2021;19(5.5):655-657 doi: $10.6004 /$ jnccn.2021.5016

The landscape of relapsed chronic lymphocytic leukemia (CLL) has changed dramatically over the past few years primarily due to changes in the frontline setting. During the NCCN 2021 Virtual Annual Conference, Jennifer R. Brown, MD, PhD, Director, CLL Center, Division of Hematologic Malignancies, and Institute Physician, Dana-Farber Cancer Institute, and Professor of Medicine, Harvard Medical School, discussed appropriate therapy for patients after relapse from various first-line treatments, including ibrutinib and venetoclax.

\section{Ibrutinib}

Ibrutinib, a BTK inhibitor, was the first targeted therapy studied in the relapsed/refractory setting. Data from a phase I study of ibrutinib demonstrated a progressionfree survival (PFS) of 32\% after 7-year follow-up, with approximately 1 in 5 patients still on the drug. ${ }^{1}$ Results of this study also showed that patients with del(17p) and del(11q) have a shorter PFS compared with those with other cytogenetic abnormalities. Furthermore, complex karyotype combined with del(17p) is significantly more adverse than del(17p) or complex karyotype alone. No difference in outcome based on IGHV mutational status has been observed.

Final results of the RESONATE trial, ${ }^{2}$ which compared ibrutinib with ofatumumab in previously treated patients with CLL who had undergone 2 to 3 prior regimens, showed a median PFS of 44 months for the ibrutinib arm in this high-risk population. Analysis by dual abnormality [TP53 and del(17p)] showed decreased PFS compared with patients without those abnormalities.

\section{Acalabrutinib}

Acalabrutinib, a highly selective next-generation BTK inhibitor, has also been studied in the relapsed/refractory setting. In the first trial to compare targeted agents headto-head, patients with relapsed/refractory CLL were randomized to acalabrutinib or investigator's choice of idelalisib + rituximab or bendamustine + rituximab. ${ }^{3}$ After a median follow-up of 22 months, acalabrutinib prolonged 18-month PFS versus investigator's choice therapy ( $82 \%$ vs $48 \%$, respectively). Interestingly, said Dr. Brown, there was no difference in PFS based on del(17p) or TP53 mutation status in the acalabrutinib arm, and no difference based on IGHV status.

There are still no head-to-head data comparing acalabrutinib and ibrutinib, but the ELEVATE-RR study has been reported to have met its primary endpoint of noninferiority for PFS. ${ }^{4}$

Zanubrutinib is also in development and is being actively studied in a largely accrued phase III trial in the relapsed setting. Data from the phase I trial with 24-month follow-up showed PFS rates of $91 \%$ in patients with relapsed/refractory disease. ${ }^{5}$

\section{Venetoclax}

Venetoclax, an inhibitor of BCL-2, depletes tumor cells much more rapidly by triggering apoptosis. In the 
MURANO study, ${ }^{6}$ patients with CLL who received a median of one prior regimen were randomized to venetoclax/ rituximab or bendamustine/rituximab. The venetoclax/rituximab arm included 6 months of rituximab and 2 years of venetoclax. With 5-year follow-up, median PFS was 54 months with venetoclax/rituximab versus 17 months with bendamustine/rituximab. Five-year overall survival also favored the venetoclax arm at $82 \%$.

According to Dr. Brown, undetectable minimal residual disease (MRD) on venetoclax has also been shown to be associated with improved PFS in several contexts.

When choosing between a BTK inhibitor and venetoclax/rituximab for a first novel agent, Dr. Brown noted that ibrutinib has longer follow-up data, and the use of a next-generation BTK inhibitor has improved the toxicity profile. In addition, a high overall response rate with venetoclax after treatment with a BTK inhibitor has been well established, but there are less data on BTK inhibition after venetoclax. Furthermore, the intense early monitoring with venetoclax favors BTK inhibition.

Conversely, high complete response rates and undetectable MRD favors the use of venetoclax/rituximab. There are also fewer long-term adverse effects with the latter combination. There is also the potential to use venetoclax/ rituximab again in patients who stop as planned and have a durable remission. Many patients prefer venetoclax/rituximab because it's a time-limited therapy, which also has the benefit of significantly less cost, said Dr. Brown.

\section{Ibrutinib + Venetoclax}

The phase II CLARITY study, which combined ibrutinib and venetoclax for patients with relapsed/refractory CLL, had a primary endpoint of MRD eradication after 12 months. ${ }^{7}$ Study results showed that rates of undetectable MRD increased progressively during the first 2 years on therapy, and provided interesting data on the relationship between the rate of disease depletion and PFS, said Dr. Brown. Most patients with rapid depletion were able to stop therapy and sustain undetectable MRD, with only 1 of 25 patients becoming positive after discontinuation. Among patients with $<2$ log depletion, however, only $20 \%$ were able to stop therapy with sustained undetectable MRD.

"Lower level of residual disease was generally associated with longer time to progression, while relapse tends to occur earlier with less depletion of disease," she observed. "Another notable element about this study is that the 3-year PFS rate was 96\%," she added. "That's very encouraging for a population of patients with relapsed/refractory CLL."

\section{MRD in General Practice}

Currently, MRD is used only for prognostic purposes with time-limited therapy. Undetectable MRD at end of treatment on the venetoclax regimen is strongly associated with the durability of remission, said Dr. Brown, but this does not apply to evaluating single-agent BTK inhibitors. "For time-limited therapies, we are very interested in performing studies to learn whether MRD can guide treatment decisions so that patients can stop treatment after achieving MRD negativity instead of having a fixed duration of therapy," said Dr. Brown. "For patients who achieve undetectable MRD but then have recurrent positivity, we'd also like to see whether they might benefit from earlier re-treatment."

\section{PI3K Inhibitors}

PI3K inhibitors are another class of drug used to treat CLL. Idelalisib + rituximab received the first approval in the relapsed/refractory setting, with a median PFS of 19.4 months in heavily pretreated patients with comorbidities. $^{8}$ "This looked encouraging," said Dr. Brown. "Unfortunately, $40 \%$ of patients discontinued after 2 years due to adverse events, including diarrhea, colitis, transaminitis, infection, and pneumonitis."

Nevertheless, patients not developing an autoimmune toxicity or an infection experience few adverse effects. Importantly, she said, combination idelalisib + rituximab is not contraindicated in cardiac or renal disease and is appropriate for use in older, pretreated patients with significant comorbidities that impact BTK inhibition (cardiac) or venetoclax tolerability (renal).

\section{Treatment After Ibrutinib}

A retrospective analysis of toxicities and outcomes for patients treated with ibrutinib showed a median PFS of 36 months, and overall survival has not been reached. ${ }^{9}$ With a median follow-up of 17 months, however, the estimated discontinuation rate on ibrutinib was $42 \%$ and median time to discontinuation was 6 months.

A real-world retrospective analysis of 683 patients with CLL demonstrated that use of venetoclax or an alternate kinase inhibitor after discontinuation on ibrutinib or idelalisib was associated with superior PFS versus chemoimmunotherapy or monoclonal antibodies. ${ }^{10}$ Median PFS on either venetoclax or alternate kinase inhibitor has not yet been reached but follow up is still relatively short.

For patients who experience progression on ibrutinib, however, Dr. Brown called the data "more worrisome." Prospective data evaluating venetoclax in CLL patients demonstrated an overall response rate of $65 \%$, but only $9 \%$ of patients experienced a complete response, ${ }^{11}$ which is "lower than that seen in patients who have not been previously treated with ibrutinib," said Dr. Brown. Median PFS was approximately 2 years.

\section{Treatment After Venetoclax}

For patients whose disease relapses after venetoclax/rituximab, the data are encouraging, said Dr. Brown, who 
noted that $48 \%$ of patients re-treated thus far from the MURANO study received venetoclax, whereas $27 \%$ received a BTK inhibitor. ${ }^{12}$ Response rates to subsequent venetoclax-based therapy were high $(72 \%)$, and to subsequent BTK inhibitor-based therapy were even higher (100\%).

Patients who experience disease relapse after both BTK inhibition and venetoclax should be encouraged by recent data on LOXO-305, a potent and selective noncovalent inhibitor of wild-type and C481-mutant BTK. In patients with CLL treated with LOXO-305, results of a phase I/II study showed an overall response rate of $63 \%$, which improved to $86 \%$ for patients on treatment for $>10$ months. ${ }^{13}$

"Responses occurred regardless of whether patients received prior BTK inhibition or prior venetoclax, and regardless of whether they discontinued their BTK inhibitor for progression or for toxicity," said Dr. Brown. "Additionally, the rate of grade 3 and 4 adverse events was extremely low, so this drug looks quite encouraging, although follow-up is still short."

Disclosures: Dr. Brown has disclosed receiving consulting fees from AbbVie, Inc., Acerta Pharmaceuticals LP, AstraZeneca Pharmaceuticals LP BeiGene, Catapult Therapeutics, Eli Lilly and Company, Genentech, Inc., Juno Therapeutics, Inc./Celgene Corporation, MEl Pharma Inc., Novartis Pharmaceuticals Corporation, Pfizer Inc., and Rigel Pharmaceuticals, Inc.; receiving grant/research support from Loxo Oncology, Inc., TG Therapeutics, and Verastem, Inc.; and receiving other financial benefit from MorphoSys AG

Correspondence: Jennifer R. Brown, MD, PhD, Dana-Farber Cancer Institute, 450 Brookline Avenue, Boston, MA 02115

Email: jbrown2@partners.org

\section{References}

1. O'Brien S, Furman RR, Coutre $S$, et al. Single-agent ibrutinib in treatment-naïve and relapsed/refractory chronic lymphocytic leukemia: a 5-year experience. Blood 2018;131:1910-1919.

2. Munir T, Brown JR, O'Brien S, et al. Final analysis from RESONATE: up to six years of follow-up on ibrutinib in patients with previously treated chronic lymphocytic leukemia or small lymphocytic lymphoma. Am J Hematol 2019;94:1353-1363.

3. Ghia P, Pluta A, Wach M, et al. ASCEND: phase III, randomized trial of acalabrutinib versus idelalisib plus rituximab or bendamustine plus rituximab in relapsed or refractory chronic lymphocytic leukemia. J Clin Oncol 2020;38:2849-2861.

4. Calquence met primary efficacy endpoint in head-to-head trial against ibrutinib in chronic lymphocytic leukaemia [press release]. Wilmington, DE: Business Wire; January 25, 2021.

5. Tam CS, Trotman J, Opat S, et al. Phase 1 study of the selective BTK inhibitor zanubrutinib in B-cell malignancies and safety and efficacy evaluation in CLL. Blood 2019;134:851-859.

6. Kater $\mathrm{AP}, \mathrm{Wu} \mathrm{JQ}$, Kipps $\mathrm{T}$, et al. Venetoclax plus rituximab in relapsed chronic lymphocytic leukemia: 4-year results and evaluation of impact of genomic complexity and gene mutations from the MURANO phase III study. J Clin Oncol 2020;38:4042-4054.

7. Hillmen $\mathrm{P}$, Rawstron $A C$, Brock $K$, et al. Ibrutinib plus venetoclax in relapsed/refractory chronic lymphocytic leukemia: the CLARITY study [published correction appears in J Clin Oncol 2020;38:1644]. J Clin Oncol 2019;37:2722-2729.

8. Brown JR, Byrd JC, Coutre SE, et al. Idelalisib, an inhibitor of phosphatidylinositol 3-kinase $\mathrm{p} 110 \delta$, for relapsed/refractory chronic lymphocytic leukemia. Blood 2014;123:3390-3397.

9. Mato AR, Nabhan C, Thompson MC, et al. Toxicities and outcomes of 616 ibrutinib-treated patients in the United States: a real-world analysis. Haematologica 2018;103:874-879.

10. Mato AR, Hill BT, Lamanna N, et al. Optimal sequencing of ibrutinib, idelalisib, and venetoclax in chronic lymphocytic leukemia: results from a multicenter study of 683 patients. Ann Oncol 2017;28:1050-1056.

11. Jones JA, Mato AR, Wierda WG, et al. Venetoclax for chronic lymphocytic leukaemia progressing after ibrutinib: an interim analysis of a multicentre, open-label, phase 2 trial. Lancet Oncol 2018;19:65-75

12. Harrup RA, Owen C, D'Rozario J, et al. Efficacy of subsequent novel targeted therapies, including repeated venetoclax-rituximab (VenR) in patients (Pts) with relapsed/refractory chronic lymphocytic leukemia (R/R $\mathrm{CLL})$ previously treated with fixed-duration VenR in the Murano study. Blood 2020;136(Suppl 1):44-45

13. Mato AR, Pagel JM, Coombs CC, et al. LOXO-305, a next generation, highly selective, non-covalent BTK inhibitor in previously treated CLL/ SLL: results from the phase $1 / 2$ BRUIN study [abstract]. Presented at the 62nd ASH Annual Meeting and Exposition; December 5-8, 2020. Abstract 542. 